\title{
PALMPRINT VERIFICATION USING COMPLEX WAVELET TRANSFORM
}

\author{
Lei Zhang ${ }^{1}$, Zhenhua Guo ${ }^{1}$, Zhou Wang $^{2}$ and David Zhang ${ }^{1}$ \\ ${ }^{1}$ Biometrics Research Center, Dept. of Computing, The Hong Kong Polytechnic Univ., Hong Kong \\ ${ }^{2}$ Dept. of Electrical Engineering, The University of Texas at Arlington, Arlington TX 76019, USA
}

\begin{abstract}
Palmprint is a unique and reliable biometric characteristic with high usability. With the increasing demand of automatic palmprint authentication systems, the development of accurate and robust palmprint verification algorithms has been attracting a lot of interests. The relative translation, rotation and distortion between two palmprint images will introduce much error in palmprint matching. However, an accurate registration of palmprint images is too time-consuming. In this paper, we propose a modified complex wavelet structural similarity index (CW-SSIM) to compute the matching score and hence identify the input palmprint. Since CW-SSIM is robust to translation, small rotation and distortion, a fast rough alignment of palmprint images is sufficient. CW-SSIM is also insensitive to luminance and contrast changes. Our experimental results show that the proposed scheme outperforms the state-of-theart methods by achieving a higher genuine acceptance rate and a lower false acceptance rate simultaneously.
\end{abstract}

Index Terms - Biometrics, palmprint, complex wavelet transform, similarity measurement

\section{INTRODUCTION}

Automatic authentication using biometric characteristics is playing a more and more important role in security. Biometric approaches recognize the identity of a person with certain physiological characteristics, including the extensively studied fingerprints, facial features, iris, speech, hand geometry, etc. In the past several years, a new biometric technique, palmprint recognition, has been proposed [1-5]. The palmprint recognition system has many merits. For example, it is reliable, low cost and userfriendly.

Similar to fingerprint and iris, palmprint is one of the most reliable means in personal identification because of its stability and uniqueness. Different to fingerprint and iris, palmprint-based identifier is more user-friendly. Referring to Fig. 1 (a), in the palmprint identification system developed by the Biometrics Research Centre, The Hong Kong Polytechnic Univ., a low-cost CCD camera is used to capture the palmprint image at a very low resolution (75 dpi). Fig. 1(b) shows a typical palmprint image captured by the system.

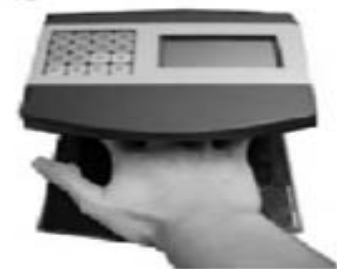

(a)

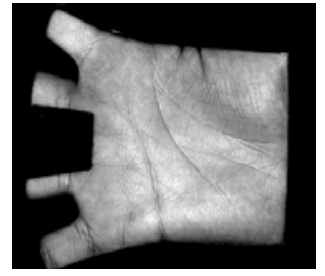

(b)
Figure 1. (a) The palmprint image acquisition system. (b) A typical palmprint image.

The main types of features in the palmprint are principal lines, wrinkles and creases. There are usually three principal lines in a palmprint: the heart line, the head line, and the life line. These lines vary little over time, and their shapes and locations on the palm are the most important physiological features for individual identification. Wrinkles are much thinner than the principal lines and much more irregular. Creases are detailed textures, like the ridges in a fingerprint, all over the palmprint. Creases can only be captured using high resolution cameras. With the low-resolution palmprint image, the principal lines and thick wrinkles will be exploited for verification. Many palmprint verification schemes [2-5] have been proposed, including Gabor filtering, wavelet, etc.

One important issue in palmprint verification schemes is an accurate registration of the input palmprint. To reduce the error caused by palm rotation and translation, a peg is employed in the palmprint system (referring to Fig. 1) to fix the hand and a ROI (region of interest) preprocessing step (referring to section 3.1) is used to further adjust the palmprint. This operation roughly aligns the palmprint image and it does not reduce the effect of palmprint distortion. An accurate registration of the input palmprint with the database, however, is unrealistic because it is too time-consuming.

The objective of this paper is to find a high accuracy (high genuine acceptance rate and low false acceptance rate) palmprint verification scheme without accurate image 
registration. To this end, a recently proposed image similarity metric, complex wavelet structural similarity index (CW-SSIM) [6], is employed and modified to compare the input palmprint with the one in the database. CW-SSIM transforms the pair of images to be compared into complex wavelet domain and then uses a sliding window to compute the local similarity. Though CW-SSIM index was originally proposed to measure the image quality, it has good properties which are desired for palmprint matching. For instance, it is insensitive to spatial translation, small rotation and distortion. It is also robust to luminance and contrast change. All these properties make it a good candidate to work as a palmprint verification metric.

The rest of the paper is as follows. Section 2 briefly introduces the CW-SSIM index. Section 3 describes the proposed palmprint verification scheme using CW-SSIM. Section 4 presents the experimental results and Section 5 gives the conclusion.

\section{THE COMPLEX WAVELET STRUCTURAL SIMILARITY INDEX}

In [6], Wang et al proposed a complex wavelet structural similarity (CW-SSIM) index for evaluating image quality. CW-SSIM was developed to overcome the drawbacks of spatial domain structural similarity (SSIM) index previously proposed by Wang et al [7]. SSIM index is highly sensitive to geometrical distortions such as translation, scaling, rotation, or other misalignments. The use of the complex wavelet transform is motivated in two ways. First, Fourier phase carries more important information about image structures than Fourier magnitude and wavelet phase has been successfully applied in image processing applications [9]. Second, small translations, scalings and rotations lead to consistent, describable phase changes in the complex wavelet domain.

Consider a mother wavelet $w(u)=g(u) e^{j \omega_{c} u}$, where $\omega_{c}$ is the center frequency of the modulated band-pass filter and $g(u)$ is a slowly varying and symmetric function. The family of wavelets are dilated and translated versions of $w(u)$ :

$$
w_{s, p}(u)=\frac{1}{\sqrt{s}} w\left(\frac{u-p}{s}\right)=\frac{1}{\sqrt{s}} g\left(\frac{u-p}{s}\right) e^{j \omega_{c}(u-p) / s}
$$

where scale factor $s \in R^{+}$and translation factor $p \in R$. The continuous complex wavelet transform of a given real signal $x(u)$ is [8]:

$$
X(s, p)=\frac{1}{2 \pi} \int_{-\infty}^{\infty} X(\omega) \sqrt{s} G\left(s \omega-\omega_{c}\right) e^{j \omega p} d \omega
$$

where $X(\omega)$ and $G(\omega)$ are the Fourier transforms of $x(u)$ and $g(u)$, respectively. The discrete wavelet coefficients are sampled versions of the continuous wavelet transform.

To compute the CW-SSIM index of two images, we first compute the complex wavelet transform of them. Denote by $\mathbf{c}_{x}=\left\{c_{x, i} \mid i=1,2, \cdots N\right\} \quad$ and $\quad \mathbf{c}_{y}=\left\{c_{y, i} \mid i=1,2, \cdots N\right\}$ the complex wavelet coefficients of two images. The CW-SSIM index of $\mathbf{c}_{x}$ and $\mathbf{c}_{y}$ is computed as [6]:

$$
\widetilde{S}\left(\mathbf{c}_{x}, \mathbf{c}_{y}\right)=\frac{2\left|\sum_{i=1}^{N} c_{x, i} c_{y, i}^{*}\right|+K}{\sum_{i=1}^{N}\left|c_{x, i}\right|^{2}+\sum_{i=1}^{N}\left|c_{y, i}\right|^{2}+K}
$$

Here $c^{*}$ denotes the complex conjugate of $c$ and $K$ is a small positive constant. It has been shown that the CW-SSIM index is insensitive to luminance and contrast changes as well as small translation, rotation and distortion.

\section{PALMPRINT VERIFICATION WITH MODIFIED CW-SSIM INDEX}

This section describes the proposed palmprint verification algorithm using a modified CW-SSIM index as a metric. We first extract from the original palmprint images a region of interest (ROI). This process can also roughly align the palmprint images. The complex wavelet transform will be performed on the ROIs of the palmprint images.

\subsection{Determination of ROI}

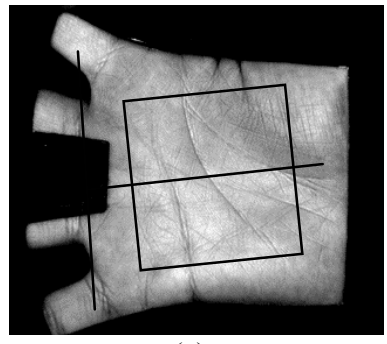

(a)

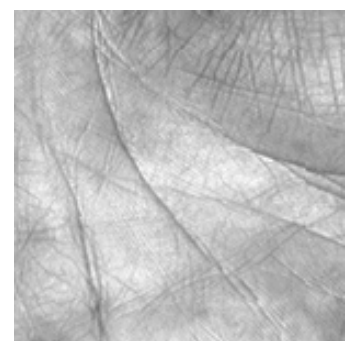

(b)
Figure 2. (a) The determination of ROI. (b) A cropped ROI image of the palmprint image in (a).

Once the palmprint is captured, it is processed to get the region of interest (ROI), which is a $128 * 128$ area, for feature extraction and identity recognition. This process will also reduce, to some extent, the effect of rotation and translation of the hand. Please refer to [2] for the detailed ROI determination process. Figure 2 illustrates a ROI image cropped from the original palmprint image.

\subsection{Modified local CW-SSIM index}

After determining the ROI of the palmprint, we apply the complex wavelet transform to it. In implementing the complex wavelet transform, we use a complex version of the "steerable pyramid" transform [10], which is a type of redundant wavelet transform that avoids aliasing in subbands. We decompose the ROI image into 3 scales with 16 orientations at each scale. To reduce the storage space and computation load in matching, only the wavelet coefficients at the $3^{\text {rd }}$ scale are used for verification. In Fig. 
3, we show two palmprint images from two persons. The complex wavelet transform images of them in one orientation at scale 3 are shown in Fig. 4 with real and imaginary parts being displayed separately.

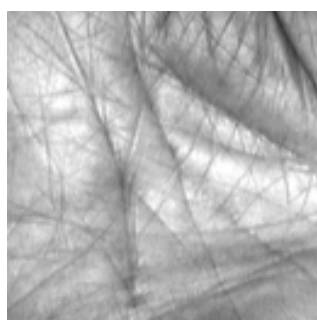

(a)

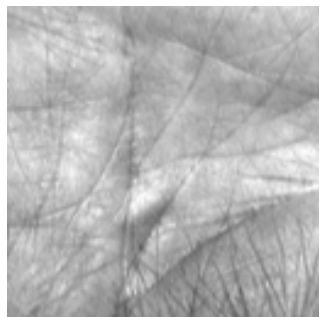

(b)
Figure 3. Two palmprint ROI images from two persons.

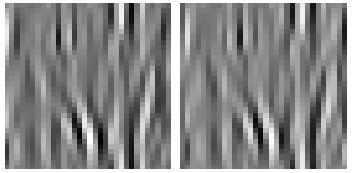

(a)

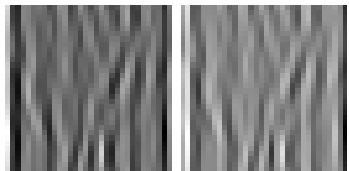

(b)
Figure 4. The complex wavelet coefficients of the two images in Fig. 3. Left is the real part and right is the imaginary part.

We can rewrite (3) as

$$
\widetilde{S}\left(\mathbf{c}_{x}, \mathbf{c}_{y}\right)=\frac{2 \sum_{i=1}^{N}\left|c_{x, i}\right|\left|c_{y, i}\right|+K}{\sum_{i=1}^{N}\left|c_{x, i}\right|^{2}+\sum_{i=1}^{N}\left|c_{y, i}\right|^{2}+K} \cdot \frac{2\left|\sum_{i=1}^{N} c_{x, i} c_{y, i}^{*}\right|+K}{2 \sum_{i=1}^{N}\left|c_{x, i}\right|\left|c_{y, i}\right|+K}
$$

The first component in the right hand of (4) is completely determined by the magnitudes of the coefficients. The maximum value 1 is achieved if and only if $\left|c_{x, i}\right|=\left|c_{y, i}\right|$ for all values of $i$. The second component is fully determined by the consistency of phase changes between $\mathbf{c}_{x}$ and $\mathbf{c}_{y}$. It achieves the maximum value 1 when the phase difference between $c_{x, i}$ and $c_{y, i}$ is a constant for $i$. The second component is a better measure of image structural similarity than the first one because the structural information of local image features is mainly contained in the relative phase patterns of the wavelet coefficients and a constant phase shift of all coefficients does not change the structure of local image features. With these considerations, we only use the second component of (4) to verify the similarity between palmprints. The modified CW-SSIM is defined as

$$
\tilde{S}_{d}\left(\mathbf{c}_{x}, \mathbf{c}_{y}\right)=\frac{\left|\sum_{i=1}^{N} c_{x, i} c_{y, i}^{*}\right|+K}{\sum_{i=1}^{N}\left|c_{x, i}\right|\left|c_{y, i}\right|+K}
$$

In calculating the local similarity of two wavelet coefficient images, we move a sliding window $\mathbf{w}$, whose size is $l \times l$, across each subband. At position $(n, m)$, we have a set of wavelet coefficients within the window and denote it as $\mathbf{w}_{(n, m)}=\left\{w_{i} \mid i=1,2, \cdots, l^{2}\right\}$. For two sets of wavelet coefficients $\mathbf{w}_{(n, m)}^{a}$ and $\mathbf{w}_{(n, m)}^{b}$ from two palmprints, the local CW-SSIM at $(n, m)$ is $S(n, m)=\tilde{S}_{d}\left(\mathbf{w}_{(n, m)}^{a}, \mathbf{w}_{(n, m)}^{b}\right)$. The overall similarity of the two palmprints, denoted by $s$, can be estimated as the average of all local CW-SSIM $S(n, m)$ across all orientations. If the similarity index $s$ of two palmprint images is higher than a preset threshold, we say they are from the same person.

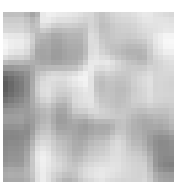

(a)

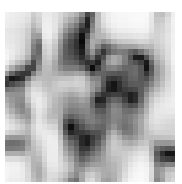

(b)
Figure 5. The normalized gray-scale similarity map between the palmprint images in Fig. 3. (a) is the result by using the first component in (4) and (b) is the result by using the second component in (4).

To further illustrate why we choose measurement (5) but not (4), let's compute the similarities of the two palmprint images in Fig. 3 by using the first and second components in (4) respectively. The left and right images in Fig.5 are the outputs using the first and second components in (4) respectively. We can clearly see that the left similarity map is much brighter than the right one. This means that two different palmprint images are hard to be discriminated by the first components in (4), which is verified by our experiments too.

\section{EXPERIMENTS}

Our test palmprint database contains 600 grayscale images collected from 100 different palms, six samples each palm. Those palmprint images were collected in two sessions with an average time interval of 69 days.

To obtain the verification accuracy of our method, each palmprint image is matched with all the other palmprint images in the database. A match is counted as correct if the two palmprint images are from the same palm; otherwise, the match is counted as incorrect. The total number of matches is $600 \times 599 / 2=179,700$, and among them there are $600 \times 5 / 2=1,500$ correct matchings. In Fig. 6 , we plot the curve of Genuine and Impostor similarity distribution for the proposed method.

The competitive coding scheme in [3], one of the most successful palmprint verification schemes, is used for comparison with the proposed CW-SSIM scheme. Fig. 7 plots the Receiver Operating Characteristic (ROC) curves (the genuine acceptance rate against the false acceptance rate for all possible operating points) for the two methods. From Fig. 7 we can see that the proposed method can operate at genuine acceptance rate of $99.2 \%$ while the corresponding false acceptance rate is $1 \times 10^{-5}$. The equal error rate (EER) of competitive coding is 0.1988 , while that of our method is 0.0684 , only one-third of the former. 


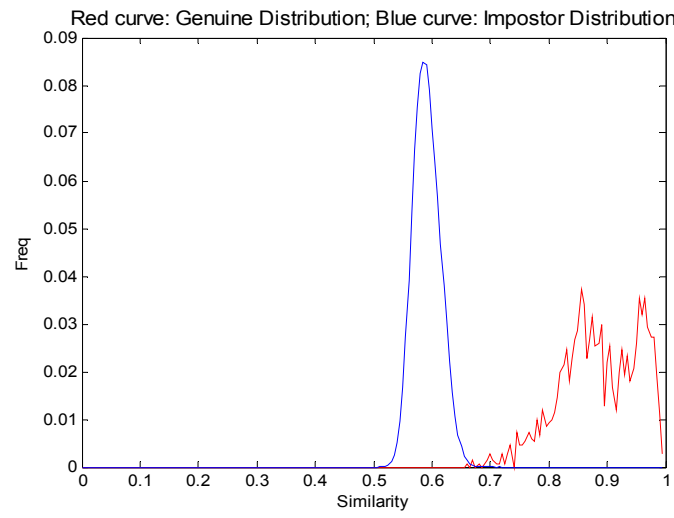

Figure 6. Similarity Distribution.

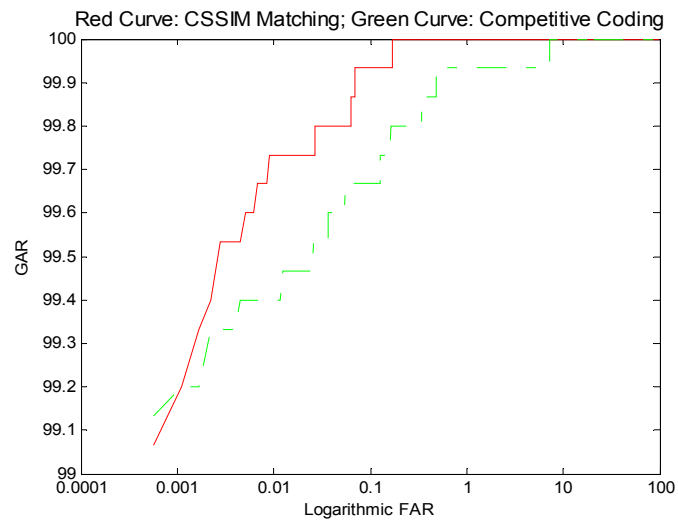

Figure 7. ROC of CW-SSIM \& Competitive Coding.

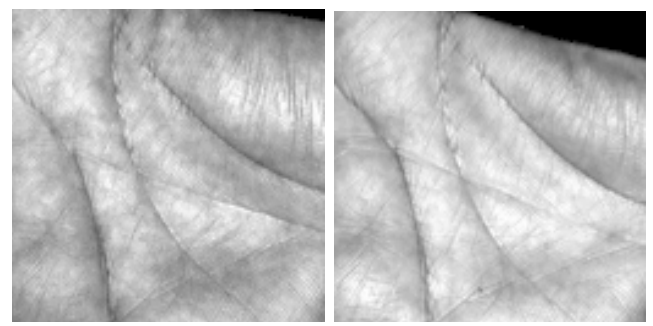

Figure 8. Two palmprints from the same person. The similarity between the two images is 0.7129 by using CW-SSIM while it is only 0.6092 by utilizing Competitive Coding Scheme.

One of the advantages of CW-SSIM index is its robustness to image distortions. Fig. 8 shows an example. The two palmprints are from the same person and the second one has some distortion. The distortion was not fully reduced in the ROI determination process and it makes the competitive coding scheme fail. The proposed CW-SSIM scheme, however, is robust to this distortion and it correctly identifies that the two palmprints are from the same person.

\section{CONCLUSION}

We proposed a palmprint similarity measurement by using complex wavelet transform. The image local structure information is hidden in the relative phase patterns of the complex wavelet coefficients and a constant phase shift of all coefficients does not change the structure of local image features. Therefore, the proposed CW-SSIM metric is robust to translation, small rotation and distortion. Our experiments validated that it achieves higher genuine acceptance rate and lower false acceptance rate than the competitive coding method, which is one of the best palmprint verification schemes. One drawback of the proposed scheme is its relatively big memory requirement to store the wavelet coefficients. How to find a more compact representation of the information hidden in complex wavelet coefficients to reduce the storage space requirement is the main task of our future work.

\section{REFERENCES}

[1] D. Zhang, Palmprint Authentication, Kluwer Academic Publishers, USA, 2004.

[2] D. Zhang, W.K. Kong, J. You and M. Wong, "On-line palmprint identification", IEEE Trans. on Pattern Analysis and Machine Intelligence, vol. 25, pp. 1041-1050, Sept. 2003.

[3] A. Kong and D. Zhang, "Competitive coding scheme for palmprint verification", Proc. of International Conference on Pattern Recognition, 2004, vol. 1, pp. 520-523, Cambridge, UK.

[4] L. Zhang and D. Zhang, 2004, "Characterization of palmprints by wavelet signatures via directional context modeling", IEEE Trans. on SMC-B, vol. 34, pp. 1335-1347, June 2004.

[5] W.K. Kong, D. Zhang and W. Li, 2003, "Palmprint feature extraction using 2-D Gabor filters", Pattern Recognition, vol. 36, pp. 2339-2347, Oct. 2003.

[6] Z. Wang and E.P. Simoncelli, "Translation insensitive image similarity in complex wavelet domain," in IEEE International Conference on Acoustics, Speech, and Signal Processing, 2005, vol. 2, pp. 573-576.

[7] Z. Wang, A. C. Bovik, H. R. Sheikh, and E. P. Simoncelli, "Image quality assessment: From error visibility to structural similarity," IEEE Trans. Image Processing, vol. 13, pp. 600-612, Apr. 2004.

[8] Z. Wang and E. P. Simoncelli, "Local phase coherence and the perception of blur," in Adv. Neural Information Processing Systems (NIPS03), vol. 16, (Cambridge, MA), MIT Press, May 2004.

[9] J. Portilla and E. P. Simoncelli, "A parametric texture model based on joint statistics of complex wavelet coefficients," Int'l $J$ Computer Vision, vol. 40, pp. 49-71, 2000.

[10] E. P. Simoncelli, W. T. Freeman, E. H. Adelson, and D. J. Heeger, "Shiftable multi-scale transforms," IEEE Trans Information Theory, vol. 38, pp. 587-607, Mar 1992. 